\title{
Is it time to replace the star ratings adherence measures?
}

\section{SUMMARY}

Measures of medication adherence to noninsulin diabetes medications (NIDM), statins, and renin angiotensin system antagonists (RASA) have been continuously used in the star ratings program since 2012 . Adherence to these treatments is undoubtedly important. However, the consistent use of these measures in the star ratings program over time has limitations.

The purpose of this Viewpoints article is to highlight concerns associated with the current star ratings adherence measures and to offer proposals for the improvement of the adherence measurement in the program. This scoping review outlines concerns with the validity of the 3 star ratings adherence measures. In addition, star ratings data are used to describe payment thresholds for the 3 adherence measures over time to describe concerns associated with their use.

Since 2012, there has been significant growth in the proportion of patients considered adherent in the star ratings program. Rates of adherence in a 2-star plan for NIDM (79\%), RASA agents ( $83 \%$ ), and statins (79\%) now exceed what is commonly reported in the clinical literature. For a plan to achieve a rating of 5 stars, more than $88 \%$ of patients must be adherent to each measure. These rates suggest a ceiling effect and a reduced ability to distinguish plan performance. In addition, concerns over the potential for plans to "game" these measures have been raised. The use of mail order services, 90-day prescription refill programs, and automatic refill reminders all improve the proportion of days covered measurement but may not reflect true adherence improvements.

Given potential concerns associated with the use of the existing adherence measures, it may be time to consider their replacement. One option would be to adopt a broader inventory of chronic medications to measure adherence in the program and to rotate medication categories in the program each year. It might also be time to explore measuring patient adherence to all medications that a patient uses instead of the narrow focus on the 3 existing measures.
Initiated in 2007, the Medicare Star Ratings Program measures the quality of stand-alone Medicare Part D prescription drug plans (PDPs) and Medicare Advantage plan offerings. ${ }^{1,2}$ Under this program, plans receive a composite quality rating, with highest performing plans receiving a 5 -star rating. This composite rating is composed of separate, validated metrics derived from information collected from plan audits, beneficiary reporting, plan reporting, and prescription/health claims records.

The star ratings program has direct and indirect influences on health plans. Medicare publicly reports the overall composite rating and individual plan metrics to inform beneficiaries of the quality of plans during the enrollment process. ${ }^{3,4}$ This publicly available information provides guidance to beneficiaries selecting a prescription drug plan. The effect of the star

\section{Author affiliations}

Joel F Farley, PhD, University of Minnesota College of Pharmacy, Minneapolis, and Benjamin Y Urick, PharmD, PhD, Center for Medication Optimization, University of North Carolina Eshelman School of Pharmacy, Chapel Hill.

\section{AUTHOR CORRESPONDENCE:} Joel F Farley, 612.624.9624; farl0032@umn.edu

J Manag Care Spec Pharm 2021;27(3):399-404

Copyright $\odot 2021$, Academy of Managed Care Pharmacy. All rights reserved.

ratings program on enrollment decisions is apparent in the literature, with at least 1 study reporting a 1-point increase in star ratings, leading to a 9.5 percentage point increase in the likelihood of enrolling in a plan. ${ }^{5}$ More directly, the star ratings program can influence Medicare Advantage plan payments through quality bonus payment incentives. In 2018 alone, an estimated $\$ 6.3$ billion in quality bonus payments were disbursed through the Medicare Advantage program. ${ }^{6}$ 
Among the specific measures included in the star ratings program, 3 medication adherence measures receive considerable attention, since they contribute triple weighting toward the final composite score. The 3 measures, which have been continuously used in the star ratings program since 2012, include adherence to noninsulin diabetes medications (NIDM), statin medications, and renin angiotensin system (RAS) antagonists. The focus on adherence to these treatments is not surprising given estimates that $27.2 \%$ of Medicare patients had diabetes; $57.1 \%$ had hypertension; and $40.7 \%$ had hyperlipidemia in 2017. ${ }^{7}$ Furthermore, the importance of medication adherence is well established, resulting in less frequent use of expensive health services such as inpatient and emergency department care, reduced mortality, and associated improvements in quality of life..$^{8-13}$ Overall, medication nonadherence is a significant contributor to the near $\$ 500$ billion in excess annual health spending that has resulted from inappropriate medication use in the United States. ${ }^{14}$

Although the importance of adherence is without question, the current use of the existing adherence measures in the star ratings program has important limitations. Our objective is to highlight concerns associated with the current star ratings adherence measures and offer proposals to improve adherence measurement in the program. To achieve this objective, we highlight a number of studies that have outlined concerns related to the application of the proportion of days covered (PDC) adherence measurement currently used in the star ratings program to assess quality. In addition, we examine trends in star rating adherence ratings reported by the Centers for Medicare \& Medicaid Services (CMS) from 2012 to the present and demonstrate a number of concerns that this continuous use might have on measure validity.

\section{Validity of the PDC Method}

Adherence, defined as the extent to which patients take medications as prescribed ${ }^{15}$ can be measured in a variety of ways. Although the most accurate way to measure adherence is through direct patient observation, this is invasive and simply not feasible for broad-based quality measurement. Therefore, prescription drug claims are frequently used to provide an estimate of adherence. The star ratings program uses the PDC method for calculating adherence, ${ }^{16}$ and CMS provides technical specifications for the PDC measurement in its annual guidance. ${ }^{2}$ Details related to the measurement are provided elsewhere ${ }^{16}$ However, the general process calculates the number of days covered by medication during an expected coverage period and dividing by the total days that a patient is expected to use medication during that coverage period. The length of the coverage period is the difference between the first fill and the last day of the year and covered days calculated using the days supply of prescriptions filled during this period. Additional adjustments are made for overlapping supply and institutionalized stays. Finally, the application of PDC in the star ratings measures involves dichotomizing patients as either adherent $(\mathrm{PDC} \geq 80 \%)$ or nonadherent $(\mathrm{PDC}<80 \%)$. The proportion of patients meeting this adherence threshold serves as the basis for assignment of a plan's star rating. There is some debate about the clinical meaningfulness of the $80 \%$ adherence threshold ${ }^{17}$ Regardless, the current incentive for plans participating in the star ratings program is to obtain this threshold for each of its members.

It is important to note that PDC is an approximation of true medication adherence, and true adherence is not possible to verify without direct patient observation. However, claims-based measures, such as PDC, have been shown to correlate well with other more direct measures of patient consumption, such as the use of medication event monitoring system caps, which measure how often a patient opens a pill bottle once a medication is dispensed. ${ }^{18}$ In addition, as previously noted, numerous studies have shown a correlation between adherence through claim-based measures and health outcomes. ${ }^{9-12}$ Therefore, while PDC is an intermediary outcome of clinical benefit, it is thought to be a good correlate of other outcomes, such as patient health and health service utilization (e.g., hospital admission and emergency room visit rates).

\section{Validity of PDC Measure Scoring}

CMS publishes annual cut-points for each star ratings quality measure to guide plans on performance standards. ${ }^{4}$ Figure 1 shows the cut-points from 2012 to 2020 that standalone PDPs and Medicare Advantage health plans must meet for each of the 3 adherence measures. Reviewing these data, there is a clear increase in the percentage of patients meeting or exceeding the $80 \%$ adherence threshold for each measure. In fact, for PDPs, the 2020 cut-point to achieve a 2-star rating is equal to or greater than the 2012 cut-point rating that assigned a plan a 5-star rating. This increase in rates across all cut-points suggests an emphasis by plans to improve member adherence.

However, there is some skepticism that the improvements reflect true patient adherence improvement and not merely improvements in PDC scores. For example, the proportion of patients that must be adherent (defined as a $\mathrm{PDC} \geq 80 \%$ ) in even a 2 -star plan for diabetes (79\%), RASA agents (83\%), and statins (79\%) now exceeds what is commonly thought to be the true rate of adherence in patients 


\section{FIGURE 1 Trends in Cut-Points for Adherence Measures in the Medicare Star Ratings Program}
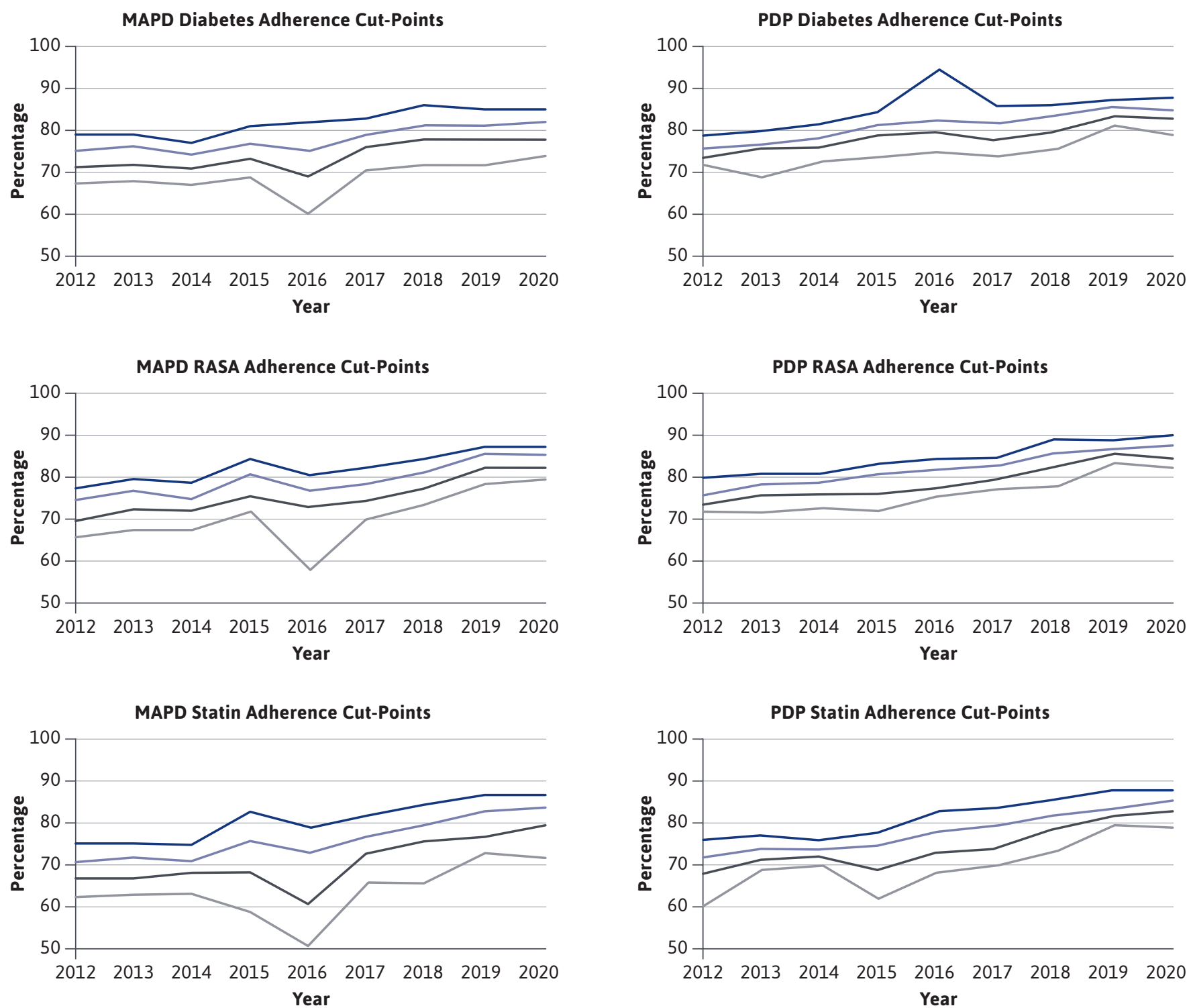

Star Rating

4

$3 \longrightarrow 2$

$M A P D=$ Medicare Advantage Prescription Drug plan; $P D P=$ Medicare Part D Prescription Drug Plan; RASA= renin angiotensin system antagonist (e.g., angiotensin receptor blockers and angiotensin-converting enzyme inhibitors). 
with these conditions. To illustrate, several studies have documented rates of medication adherence across different chronic health conditions. A recent meta-analysis comparing health outcomes between adherent and nonadherent diabetes patients across 8 different studies found that $38 \%$ of patients were not adherent to treatment. ${ }^{8}$ Similarly, a meta-analysis of medication adherence across several classes of medication, including antihypertensives, aspirin, and statins in patients with cardiovascular conditions, suggested a $57 \%$ rate of adherence. ${ }^{19}$ As rates of adherence reported in the star ratings program depart from that found in the clinical literature, concern about the validity of the reported star ratings adherence rates arises.

An additional concern associated with the increasing trend in cut-points needed to achieve higher star ratings is whether the measures might be approaching a ceiling. Indeed, in 2016 the 5-star cut-point for NIDM adherence was $95 \%$. Similarly, in 2020, for a plan to achieve a rating of 5 stars for diabetes, RASA, and statin medications, PDP plans must maintain adherence for $88 \%, 90 \%$, and $88 \%$ of patients, respectively. ${ }^{2}$ These ambitious targets require nearly all patients to have a PDC above $80 \%$ and leave little room for nonadherence. While higher is better for adherence, there are rational reasons, such as side effects, dosing changes, cash prescriptions, and samples, why a patient who is truly $100 \%$ adherent may have a PDC of less than $100 \%$. Therefore, an adherence goal of $100 \%$ is not a realistic expectation for any plan to achieve.

As the measures approach a potential ceiling, there is also a concern that the adherence measures might lose their ability to distinguish between high- and lowperforming plans. As one example, in 2019 the percentage of a PDP plan population that must adhere to RASA treatment to achieve a 5 -star rating was $89 \%$, while a 2 -star plan must maintain adherence for $84 \%$ or more of its population. This is a mere $5 \%$ difference across all plans. Distinguishability is one of a number of essential characteristics that must be met for a quality metric to be deemed a reliable measure of performance. ${ }^{20}$ As the PDC cut-points reach a ceiling, the margin between higher- and lower-performing plans becomes harder to delineate, resulting in an inability to discriminate plan performance and arbitrary disbursement of stars-linked bonuses.

\section{Health Plan Strategies to Improve Adherence}

The triple weighting of the adherence scores creates considerable incentives for health plans to implement policies and programs that improve adherence scores. Among the options available to plans are clinical services such as comprehensive medication reviews (CMRs) in the Medicare medication therapy management program. Interestingly, rates of CMR completion was adopted as an independent star ratings metric in 2016. It should be noted that CMR completion rates in the star ratings program have not been shown to correlate well with plan-level medication adherence scores. ${ }^{21}$ This suggests that CMRs may not be a primary driver of rising adherence rates observed in the star ratings program.

Other strategies to improve adherence are often less clinically focused and instead emphasize reducing barriers to filling medication. Some programs focus on targeting patients who are adherent and at risk of falling below the $80 \%$ threshold or are below the $80 \%$ threshold and can be brought above it. ${ }^{22}$ While minor changes in adherence can greatly affect a measure relying on a dichotomous cut-point, one may wonder whether this small difference has an equally large effect on true care outcomes. For example, shifting patients to 90-day prescriptions reduces the number of times a patient must visit the pharmacy to fill a medication. This has an added benefit in the PDC metric by reducing the number of times uncovered days between prescription fills can be observed in the PDC. ${ }^{23}$ Advocating for patients to enroll in scheduled refill programs further reduces the burden on patients to remember to order refills. ${ }^{24}$ Finally, shifting patients to mail order delivery reduces the burden associated with the need to travel to the pharmacy for each medication fill.

Given that PDC measures patterns of prescription fills and not actual consumption, a question arises regarding whether the administrative strategies, such as 90-day prescriptions and scheduled refill programs, used to improve adherence scores represent true improvements in actual patient adherence. With the monetary benefits associated with better star ratings, the potential for "gaming" of these metrics cannot be discounted.

\section{Should the Star Ratings Adherence Measures be Replaced?}

Assuming that the rates of medication adherence reported in the star ratings program are accurate, the inclusion of medication adherence as a core measure in the program represents a significant clinical accomplishment for the Medicare program. The resulting increase in medication adherence through adoption in the star ratings program exceeds clinical improvements commonly reported from 
the adoption of other programs. For example, the majority of studies that have examined waiving copayments for patients using chronic medications under value-based insurance design initiatives show adherence improvements of $5 \%$ or less. ${ }^{25}$ Therefore, if the program has succeeded for these 3 classes of medications, and the measures are approaching their reasonable maxima, it may be time to ask whether the adherence measures used in the star ratings program should be expanded or potentially replaced with new measures that focus on additional chronic conditions.

The continual focus in the star ratings program on adherence to 3 categories of medication comes at the expense of gains in adherence that could result from a focus on other common chronic conditions. Highly prevalent chronic conditions in which long-term medication use is common in Medicare patients include atrial fibrillation (8.4\%), heart failure (13.9\%), osteoporosis (6.4\%), ischemic heart disease (26.9\%), and arthritis (33.1\%). One option that policymakers and measure developers, such as the Pharmacy Quality Alliance (PQA), might consider is building a larger inventory of adherence measurements for use in the star ratings program. Indeed, PQA has a range of medication adherence measures, such as adherence to beta blockers, which are used long term for prevalent conditions and for which use in quality measurement programs is equally feasible.

Expanding the number of therapeutic categories in which adherence is measured would reduce the potential for plans to only emphasize adherence to the 3 current categories. The expansion of additional adherence measures could replace existing metrics that could continue to receive triple weighting. Another option would be to add adherence measures to the current 3 in use and reduce the weighting of each measure.

It should also be noted that the focus on only 3 therapeutic categories of medication may come at the expense of measuring and improving a patient's overall medication adherence behavior. Nationally, an estimated $68.9 \%$ of Medicare beneficiaries have 2 or more chronic conditions. It is further estimated that as many as $17.4 \%$ of Medicare patients have 6 or more chronic conditions. ${ }^{7}$ Another option that could be considered as a replacement to the current adherence measurements in the star ratings program is an overall measure of medication adherence in patients with multiple chronic conditions.

To explore an expanded measure of medication adherence, a proof of concept paper was recently published that evaluated a multiple medications for chronic conditions
(MMCC) measure used in a Medicaid alternative payment model for community pharmacies in North Carolina. ${ }^{26}$ In this study, patients using 4 or more medications for chronic conditions were categorized as adherent if they had a PDC of $80 \%$ or more for at least $75 \%$ of the chronic medications they used. The list of medications was more comprehensive than the current star ratings program and included 71 different therapeutic categories. In addition to MMCC adherence, this study also measured adherence for the 3 categories included in the star ratings program. Interestingly, the mean MMCC adherence rating for patients was around $30 \%$, compared with $55 \%$ of patients labeled adherent to oral diabetes medications, $46 \%$ for RASA agents, and $53 \%$ for statins.

Among patients using the 3 star ratings adherence measures that were also included in the MMCC measure, there was little correlation between the MMCC measure and star ratings adherence measures. This suggests that emphasizing adherence only to star ratings categories may come at the expense of considering the patient's overall adherence to all medications. Opportunities to focus on overall patient adherence may therefore have more value than simply focusing on the 3 chronic conditions currently emphasized in the star ratings program.

\section{Conclusions}

The 3 adherence measures used in the Medicare Star Ratings Program have been in continuous use since 2012. Throughout this period, the measures have improved considerably and may be approaching a ceiling. As the growth in plan-reported adherence departs from rates of adherence commonly reported in the clinical literature, it seems fair to question whether the reported star ratings adherence rates represent true adherence improvements or perhaps an improved understanding by drug plans of how to game adherence thresholds. If the star ratings program decides to continue using adherence measurements, it may be helpful to consider expanding the list of drugs for managing chronic conditions to reduce the potential for gaming and enhance the emphasis on adherence to other chronic conditions. The potential to measure the overall adherence of patients and all the medications they use also holds potential to reduce the burden of nonadherence on the U.S. health care system. 


\section{DISCLOSURES}

No funding supported the writing of this article. Farley has nothing to disclose. Urick reports consulting fees from Pharmacy Quality Solutions, unrelated to this work.

\section{REFERENCES}

1. Centers for Medicare \& Medicaid Services. Fact sheet-2020 Part C and D star ratings. Accessed February 8, 2021. https://www.cms. gov/Medicare/Prescription-DrugCoverage/PrescriptionDrugCovGenIn/ Downloads/2020-Star-Ratings-FactSheet-.pdf

2. Centers for Medicaid \& Medicare Services. Medicare 2019 Part C and D star rating technical notes. October 1, 2019. Accessed February 8, 2021. https://www. cms.gov/Medicare/Prescription-DrugCoverage/PrescriptionDrugCovGenIn/ Downloads/Star-Ratings-TechnicalNotes-Oct-10-2019.pdf

3. Centers for Medicare \& Medicaid Services. Find a Medicare plan. Accessed February 8, 2021. https://www.medicare. gov/plan-compare

4. Centers for Medicare \& Medicaid Services. Part C and D performance data. Accessed February 8, 2021. https://www. cms.gov/Medicare/Prescription-DrugCoverage/PrescriptionDrugCovGenIn/ PerformanceData

5. Reid RO, Deb P, Howell BL, Shrank WH. Association between Medicare Advantage plan star ratings and enrollment. JAMA. 2013;309(3):267-74.

6. Jacobson G, Damico A, Neuman T. A dozen facts about Medicare Advantage. Kaiser Family Foundation. November 13, 2018. Accessed February 8, 2021. https:// www.kff.org/medicare/issue-brief/adozen-facts-about-medicare-advantage/

7. Centers for Medicare \& Medicaid Services. Chronic conditions. Accessed February 8, 2021. https://www.cms. gov/Research-Statistics-Data-andSystems/Statistics-Trends-and-Reports/ Chronic-Conditions/CC_Main
8. Khunti K, Seidu S, Kunutsor, Davies M. Association between adherence to pharmacotherapy and outcomes in type 2 diabetes: a meta-analysis. Diabetes Care. 2017;40(11):1588-96.

9. Roebuck MC, Dougherty JS, Kaestner R, Miller LM. Increased use of prescription drugs reduces medical costs in Medicaid populations. Health Aff (Millwood). 2015;34(9):1586-93.

10. Franklin JM, Krumme AA, Tong AY, et al. Association between trajectories of statin adherence and subsequent cardiovascular events. Pharmacoepidemiol Drug Saf. 2015;24(10):1105-13.

11. Roebuck MC, Liberman JN, Gemmill-Toyama M, Brennan TA. Medication adherence leads to lower health care use and costs despite increased drug spending. Health Aff (Millwood). 2011;30(1):91-99.

12. Roebuck MC. Medical cost offsets from prescription drug utilization among Medicare beneficiaries. J Manag Care Pharm. 2014;20(10):994-95. doi: 10.18553/ jmcp.2014.20.10.994

13. Alfian SD, Sukandar H, Lestari K, Abdulah R. Medication adherence contributes to an improved quality of life in type 2 diabetes mellitus patients: a cross sectional study. Diabetes Therapy. 2016;7(4):755-64.

14. Watanabe JH, Mcinnis T, Hirsch JD. Cost of prescription drug-related morbidity and mortality. Ann Pharmacother. 2018;52(9):829-37.

15. Osterberg L, Blaschke T. Adherence to medication. N Eng J Med. 2005;353(5): 487-97.

16. Nau DP. Proportion of days covered (PDC) as a preferred method of measuring medication adherence. 2011. Accessed February 8, 2021. http://ep.yimg.com/ty/ cdn/epill/pdcmpr.pdf

17. Baumgartner PC, Haynes RB, Hersberger KE, Arnet I. A systematic review of medication adherence thresholds dependent of clinical outcomes. Front Pharmacol. 2018;9(1290):1-10. Accessed February 8, 2021. https://www.ncbi.nlm. nih.gov/pmc/articles/PMC6256123/pdf/ fphar-09-01290.pdf
18. Hansen RA, Kim MM, Song L, Tu W, Wu J, Murray MD. Adherence: comparison of methods to assess medication adherence and classify nonadherence. Ann Pharmacother. 2009;43(3):413-22.

19. Naderi SH, Bestwich JP, Wald DS. Adherence to drugs that prevent cardiovascular disease: meta-analysis on 376,163 patients. Am J Med. 2012;125(9):882-87.

20. Agency for Healthcare Research and Quality. Key questions when choosing health care quality measures. Last reviewed November 2018. Accessed February 8, 2021. https://www.ahrq.gov/ talkingquality/measures/measure-questions.html

21. Wang Y, Farley JF, Ferreri SP, Renfro CP. Do comprehensive medication review completion rates predict medication use and management quality? Res Social Adm Pharm. 2019;15(4):417-24.

22. Allazo Health. Find out how artificial intelligence can help you improve adherence by targeting your patient interventions more precisely. Accessed February 8, 2021. https://allazohealth. com/download-our-bcbs-case-study/

23. Farley JF, Urick BY, Schondelmeyer SW. Community pharmacy versus mail order: an uneven comparison [letter]. J Manag Care Spec Pharm. 2019;25(6):724-25. doi: 10.18553/jmcp.2019.25.6.724

24. Lester CA, Mott DA, Chui MA. The influence of a community pharmacy automatic prescription refill program on Medicare Part D adherence metrics. J Manag Care Spec Pharm. 2016; 22(7): 801-07. doi: 10.18553/jmcp.2016.22.7.801

25. Agarwal R, Gupta A, Fendrick AM. Value based insurance design improves medication adherence without an increase in total health care spending. Health Aff (Millwood). 2018;37(7):1057-64.

26. Farley JF, Kumar A, Urick BY. Measuring adherence: a proof of concept study for multiple medications for chronic conditions in alternative payment models. Pharmacy (Basel). 2019;7(3):81. Accessed February 8, 2021. https://www.mdpi. com/2226-4787/7/3/81/htm 